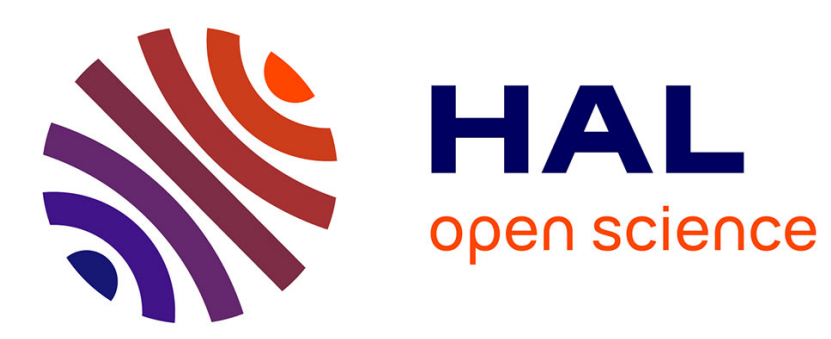

\title{
InGaN/GaN $\mu$ LED SPICE modelling with size-dependent ABC model integration
}

\author{
Anis Daami, François Olivier
}

\section{To cite this version:}

Anis Daami, François Olivier. InGaN/GaN $\mu$ LED SPICE modelling with size-dependent ABC model integration. Physics and Simulation of Optoelectronic Devices, Feb 2019, San Francisco, United States. pp.13, 10.1117/12.2509382 . cea-02941417v2

\section{HAL Id: cea-02941417 \\ https://hal-cea.archives-ouvertes.fr/cea-02941417v2}

Submitted on 29 Sep 2020

HAL is a multi-disciplinary open access archive for the deposit and dissemination of scientific research documents, whether they are published or not. The documents may come from teaching and research institutions in France or abroad, or from public or private research centers.
L'archive ouverte pluridisciplinaire $\mathbf{H A L}$, est destinée au dépôt et à la diffusion de documents scientifiques de niveau recherche, publiés ou non, émanant des établissements d'enseignement et de recherche français ou étrangers, des laboratoires publics ou privés. 

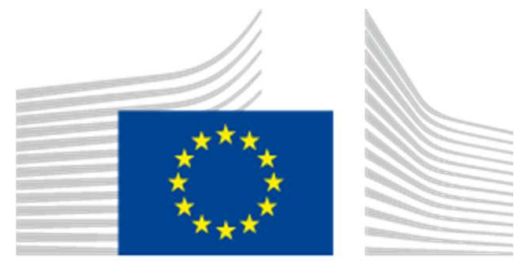

European

Commission

\begin{tabular}{|c|c|}
\hline Document Title & $\begin{array}{l}\text { InGaN/GaN } \mu \text { LED SPICE modelling with size dependent } \\
A B C \text { model integration }\end{array}$ \\
\hline Authors & $\begin{array}{l}\text { Anis Daamia , François Olivier }{ }^{\mathrm{a}} \\
\text { aUniversité Grenoble Alpes, CEA-LETI, Minatec Campus, III-V Lab, } \\
\text { Grenoble, France }\end{array}$ \\
\hline Issue date & February 26, 2019. \\
\hline Journal & $\begin{array}{l}\text { Proceedings Volume 10912, Physics and Simulation of } \\
\text { Optoelectronic Devices XXVII; 109120E (2019). }\end{array}$ \\
\hline Doi & $10.1117 / 12.2509382$ \\
\hline Acknowledgment & $\begin{array}{l}\text { This project has received funding from the Clean Sky } 2 \text { Joint } \\
\text { Undertaking under the European Union's Horizon } 2020 \text { research } \\
\text { and innovation programme under grant agreement No } 755497 .\end{array}$ \\
\hline Disclaimer & $\begin{array}{l}\text { The content of this article reflects only the author's view. } \\
\text { The Clean Sky Joint Undertaking is not responsible for any use that } \\
\text { may be made of the information it contains. }\end{array}$ \\
\hline
\end{tabular}




\title{
InGaN/GaN $\mu$ LED SPICE modelling with size dependent ABC model integration
}

\author{
Anis Daami*a, François Olivier ${ }^{\mathrm{a}}$ \\ aUniversité Grenoble Alpes, CEA-LETI, Minatec Campus, III-V Lab., Grenoble, France
}

\begin{abstract}
The need of high brightness micro-displays in portable applications dedicated to mixed and/or virtual reality has drawn an important research wave on InGaN/GaN based micro-sized light emitting diodes ( $\mu$ LEDs). We propose to use a SPICE modelling technique to describe and simulate the electro-optical behavior of the $\mu$ LED. A sub-circuit portrayal of the whole device will be used to describe current-voltage behavior and the optical power performance of the device based on the $\mathrm{ABC}$ model. We suggest an innovative method to derive instantaneously the carrier concentration from the simulated electrical current in order to determine the $\mu$ LED quantum efficiency. In a second step, a statistical approach is also added into the SPICE model in order to apprehend the spread on experimental data. This $\mu$ LED SPICE modelling approach is very important to allow the design of robust pixel driving circuits.
\end{abstract}

Keywords: InGaN/GaN, $\mu$ LED, SPICE simulations, ABC model, quantum efficiency

\section{INTRODUCTION}

Many research efforts have been carried out and a huge amount of interesting results can be found in literature dealing with the comprehension of electro-optical behavior of InGaN/GaN micro-light emitting diodes ${ }^{1,2,3}$ ( $\mu$ LEDs). Indeed, the growing interest to use these devices, especially in display and/or portable modules, is still at its very first years as no proven application can be found in the market today. An important reason is the high amount of $\mu$ LEDs needed, that have to be efficiently similar to achieve high quality $\mu$-displays $s^{4,5,6}$.

The design of these latter, is based on the combination of a pixelated array and generally a CMOS active matrix to drive the $\mu$ LEDs at their best quantum efficiency. Therefore, robust circuit design is a mandatory keystone to ensure a good functioning of these applications. Indeed process fabrication spread, even brought to a minimum, is inherent to any technology. As known and well employed in robust technologies, such as in microelectronics platforms, CMOS designs, are dependent on $\mathrm{SPICE}^{7}$ device models.

Some work has been done on devices like organic photodiodes (OPDs) or organic light emitting diodes (OLEDs) to capture through a SPICE model their electro-optical behaviors ${ }^{8,9,10}$. Some efforts towards modelling LEDs behavior can be found in literature. Nevertheless, to our knowledge, these papers generally present an analytical modelling study ${ }^{11,12,13}$ of the device or a TCAD based mode ${ }^{14}$. Therefore it has become urgent and almost mandatory to develop such models for devices like LEDs to simulate, understand and enhance the opto-electrical properties of $\mu$-displays.

Based on our latest experimental published data on blue emitting $\mu \operatorname{LEDs}^{15}{ }^{16}$, we report in this paper a complete SPICE model construction. This model aims to a complete description of the electro-optical behavior of $\mu$ LEDs through a large size range. We demonstrate the use of a simple mathematical resolution technique in the simulation core to calculate the injected carrier density from the $\mathrm{ABC}$ model equations. Consequently, the LED external quantum efficiency (EQE) calculus becomes straight forward and is emulated inside the model itself, allowing the accurate determination of the emitted optical power. Furthermore, we show the importance of the statistical approach in this type of modelling to allow the design of robust pixel driving circuits dedicated to $\mu$-display applications.

*anis.daami@cea.fr 


\section{LED OPTO-ELECTRICAL BEHAVIOR MODELLING}

\subsection{The sub-circuit approach}

We show on figure 1 the equivalent electrical circuit used to model the electrical behavior of the LED. It consists of a SPICE LEVEL 1 ideal diode (D), a series resistor (RS) and a parallel resistor (RP). This sub-circuit has two input pins representing the LED anode and cathode. The light output behavior is described by a virtual current-controlled current source $(\mathrm{F})$. This latter is biased between two nodes VN and VP that are transparent to the final user. The current value $\left(\mathrm{I}_{\mathrm{F}}\right)$ flowing through this virtual source $\mathrm{F}$ emulates the optical output power $\left(\mathrm{P}_{\mathrm{opt}}\right)$ of the LED and is defined as:

$$
I_{F}=P_{o p t}=\frac{I_{L E D}}{q} \cdot E Q E \cdot \frac{h c}{\lambda}
$$

where $\mathrm{I}_{\mathrm{LED}}, \lambda$ and EQE are respectively, the current flowing through input pins (anode to cathode), the emitted wavelength (460 $\mathrm{nm}$ in our case) and the external quantum efficiency of the LED. The reader will easily recognize the universal constants h, c and q. We will focus on the determination of EQE in section 2.3.

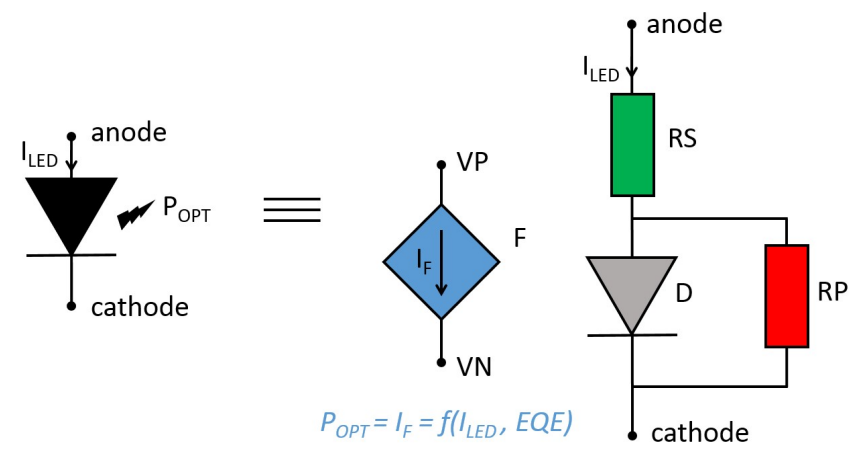

Figure 1. Equivalent electrical circuit implemented to model the LED electro-optical behavior. The components RS, RP and D are used to describe the current-voltage characteristic of the LED. The F current source is a virtual user-transparent component to mimic the optical output power of the LED.

\subsection{Electrical parameters adjustments and size effect}

Figure 2 shows current-voltage measurements carried out on vertical square-shaped LEDs of different size, ranging from $10 \mu \mathrm{m}$ to $500 \mu \mathrm{m}$. In order to adjust our SPICE model to these electrical characteristics, we have chosen to put to nil the internal series resistance of the SPICE LEVEL1 ideal diode D. The value of the implemented external resistor RS has been extracted for each LED size. The whole set permitted to derive an empirical area-dependent relationship equation as follows:

$$
R S=\frac{S 0}{S} \cdot\left(R S_{0}+R S_{V} \cdot e^{-\alpha(V-V 0)}\right)
$$

where S0 and S define the reference LED area $(500 \mu \mathrm{m}$ width), and the modelled one, respectively. Knowing that S $\leq \mathrm{S} 0$, it is evident that $\mathrm{RS}$ will present a greater value for small devices.

Furthermore, equation (2) can be interpreted as a sum of two series resistances contributions where the first term (RS $\left.\mathrm{R}_{0}\right)$ is fixed and represents the common part. The second term is voltage dependent and is seen as a non-linearity deviation of RS. Indeed, the series resistance is a representation of all the physical obstacles that injected carriers have to overcome to flow through the device. Generally, anode and cathode contacts show a deviation from ideal ohmic behavior when bias is pushed to high values. Process fabrication can also generate defects and recombination centers preventing a smooth carrier flow. For all these reasons the RS parameter value cannot be considered as a fixed value. Finally, parameter V0 is inferred as a threshold voltage from which RS non-linearity begins to act, whereas $\alpha$ is a smoothing adjustment parameter.

On the other hand, the value of the parallel resistance RP could not be adjusted for small geometries due to the limitation in our measurement instrumentation. This also demonstrates that our devices have extremely weak leakage currents at low voltage, proving a high process quality. To simplify the model and avoid any dysfunction during simulations, we have 
decided to give RP a fixed value, satisfying the lowest LED leakage current we have measured. Evidently, this choice underestimates the leakage of large geometries. Nevertheless, a light emitting device is often biased far from the leakage region above its threshold voltage, therefore this modelling hypothesis is acceptable.

Beside the RS and RP adjustments, we have tuned the saturation current (IS) and ideality coefficient ( $\eta$ ) parameters in the SPICE LEVEL1 diode model (D) to fit the low leakage and intermediate bias regions, far from the high voltage zone. Both parameter values seemed to be geometry independent.

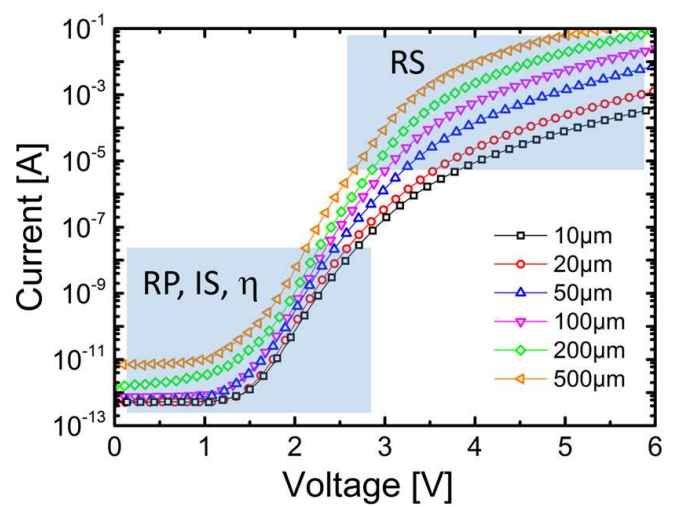

Figure 2. Current-voltage characteristics of different square LEDs. Width ranges from $10 \mu \mathrm{m}$ to $500 \mu \mathrm{m}$. Shaded areas show where the SPICE model parameters, RS, RP, IS and $\eta$ were adjusted. Note that the leakage current saturates due to a limitation in our measurement instrumentation.

\subsection{Optical quantum efficiency and ABC model}

The quantum efficiency of LEDs has thoroughly been described by the well documented ABC model in literature. We have recently suggested a slight modification to take into account size in the $\mathrm{ABC}$ model ${ }^{9}$. This modification principally affects parameter A related to the Shockley Read Hall (SRH) non-radiative recombination. Indeed, we have demonstrated that $\mathrm{A}$ is proportional to the ratio perimeter/surface $(\mathrm{P} / \mathrm{S})$ of the LED. Parameters B and $\mathrm{C}$, respectively linked to radiative and Auger non-radiative recombinations, are shown to be geometry independent. They represent the epitaxy material quality. Taking into account the electron charge density $q$ and the quantum well thickness $t$, the current density $\mathrm{J}_{\text {LED }}$ and the internal quantum efficiency (IQE) of the LED are expressed as follows:

$$
J_{L E D}=q \cdot t \cdot\left(A \cdot \frac{P}{S} \cdot n+B \cdot n^{2}+C \cdot n^{3}\right)
$$

and

$$
I Q E=\frac{B \cdot n^{2}}{A \cdot \frac{P}{S} \cdot n+B \cdot n^{2}+C \cdot n^{3}}
$$

Here, $\mathrm{n}$ is the injected carrier density flowing through the device at a given voltage. Knowing the light extraction efficiency (estimated $\mathrm{LEE} \cong 0.14$ by ray tracing simulations), the external quantum efficiency EQE is then determined from IQE in this way:

$$
E Q E=I Q E \cdot L E E
$$

We propose in this SPICE modelling methodology to integrate parameters A, B and C as input entities of the device, at the same level as its geometrical inputs, namely its perimeter $\mathrm{P}$ and surface $\mathrm{S}$. It is then obvious that if the injected carrier density $\mathrm{n}$ is known, one will not have any difficulty to estimate the quantum efficiency of the LED. Nevertheless, any SPICE simulation only allows an electrical current determination.

Our original submitted approach is then to derive instantaneously the value of $\mathrm{n}$ from the simulated electrical current. Indeed, equation (3) shows that for a given simulated current density $\mathrm{J}_{\mathrm{LED}}$, the calculus of $\mathrm{n}$ is brought to the roots 
determination of a $3^{\text {rd }}$ degree polynomial $\left(a \cdot x^{3}+b \cdot x^{2}+c \cdot x+d=0\right)$. This resolution is not straightforward as one might think. Indeed, depending on the polynomial coefficients $a, b, c$ and d different mathematical resolution methods have been proposed. We have decided to implement in the core of the LED model library, an easy and common technique called the Cardano's method ${ }^{17,18}$. This method allows a rapid calculus of all real solutions if the $3^{\text {rd }}$ degree polynomial coefficients are real, which is the case in the ABC model.

Therefore, for each applied voltage value, the current density is simulated using the adjusted electrical LED SPICE model, and the resolution of equation (3) to determine the carrier density $n$ is simultaneously carried out. Subsequently, the quantum efficiency is determined using equations (4) and (5).

\subsection{A statistical approach}

To provide a better robustness of the LED model, we have chosen to introduce a statistical modelling approach to allow the use of Monte Carlo SPICE simulations. At first approximation, the choice has been made to principally affect the injected carrier density into the LED. Therefore, we implemented Gaussian distributions on each recombination parameter A, B and C. Average parameter values have been fixed to those used in the typical model (from our latest experimental results $^{9}$ ) and a given standard deviation around $15 \%$ has been fixed for all 3 parameters. This random choice has no particular reason, except the aim to apprehend the quantum efficiency spread due to the parameters cited above. Thus, for each random Monte Carlo draw of A, B and C, the same calculus of n, IQE and EQE will be carried out as stated in section 2.3. In this special statistical configuration, it is clear that the simulated LED current will not change from a draw to another, as no variation has been introduced in the LED model parameters. Evidently, the further step would be, to evaluate the mutual effects of current variation, and recombination parameters distributions together on the LED quantum efficiency spread.

\section{SIMULATION RESULTS}

\subsection{Electrical characteristics}

We show on figure 3, the simulated current-voltage characteristics superimposed to measurement ones for the different LED sizes. It is clear that the implemented SPICE model describes well the electrical behavior, on a large voltage range. As discussed above, the current at low voltage $(0 \leq \mathrm{V} \leq 1)$ is a little bit underestimated for large geometries due to the choice of a constant value for RP accounting for LED leakage (not shown on figure 3). Despite this little discrepancy, the developed sub-circuit electrical SPICE model approach shows a high quality adjustment over the large variety of LED sizes.

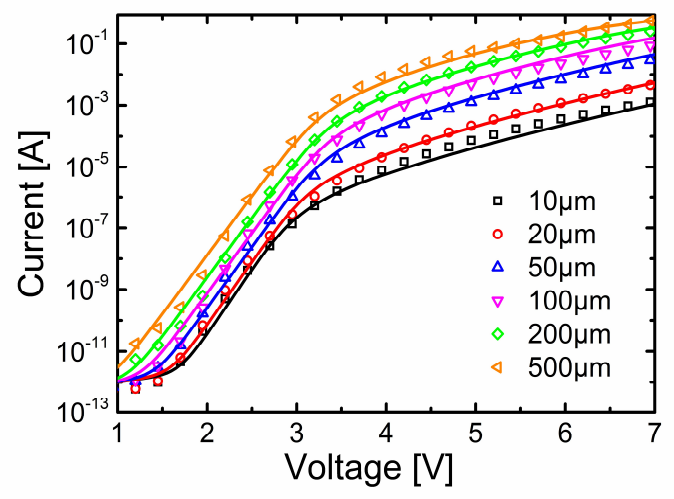

Figure 3. SPICE model simulated current-voltage characteristics (lines) versus experimental data (symbols) of different LED sizes. A good adjustment is observed on a large voltage range for all geometries. 


\subsection{Quantum efficiency}

Figure 4 shows external quantum efficiency EQE versus current density characteristics for all studied LED geometries. For the sake of clarity, experimental curves (figure 4 (a)) and simulated ones (figure 4 (b)) are not presented on the same graph. The first observation to come out is that the experimental EQE aspect is well represented by simulation. Indeed, first we observe the optical threshold shifting towards higher current density levels for small geometries. Moreover, the maximum efficiency $\mathrm{EQE}_{\max }$ drops when size diminishes as perceived on experimental data. Additionally, the common sinking of EQE known as the "droop" effect is also present on simulated graphs. Yet, looking closely to figure 4, some differences exist between measurements and simulations. Indeed the EQE optical threshold appears at lower current densities for experimental data. Besides, the $\mathrm{EQE}_{\max }$ drop versus $\mathrm{LED}$ size is somewhat exaggerated in the simulated $\mathrm{EQE}$ graphs. These differences are mainly due to the $\mathrm{ABC}$ model hypothesis, assuming a current-density $\mathrm{J}_{\mathrm{LED}}$ relationship to carrier density $\mathrm{n}$ expressed as stated in equation (3), which in reality is an empirical model based approximation. Nevertheless, for SPICE simulations it is sufficient to have the essence of the electro-optical behavior of InGaN/GaN based LEDs.

Another point worth noting is the break appearing on simulated curves whatever the LED size. This is inherent to the resolution method we have used to determine the carrier density n. Indeed, Cardano's method for the roots determination of a $3^{\text {rd }}$ degree polynomial, is highly dependent on the polynomial coefficients. This dependence implies 2 kinds of calculus subject to coefficient values. Knowing that one coefficient is directly related to current density $\mathrm{J}_{\mathrm{LED}}$, this break shows the transit between both styles of the polynomial root resolution.
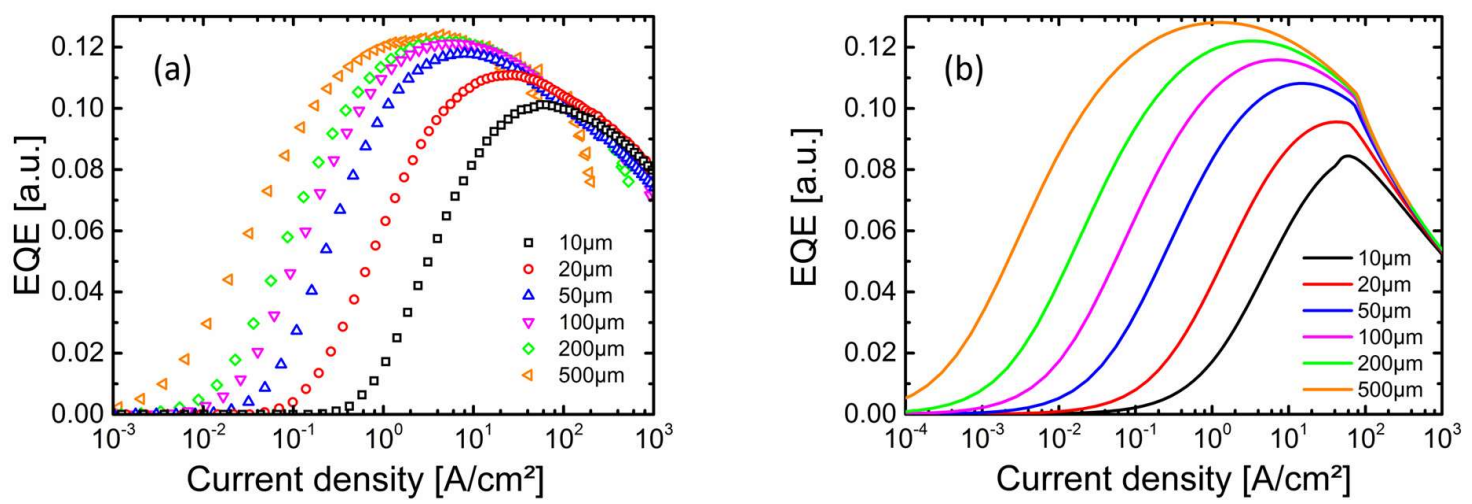

Figure 4. External quantum efficiency versus current density for different LED sizes: experimental data behavior shown in symbols (a) are well described by the simulated SPICE model (b). Some differences are yet observable between measurements and simulation, due to the $\mathrm{ABC}$ model approximation on the LED current modelling.

\subsection{Spread and recombination parameters effect}

To apprehend the effect of recombination mechanisms on the LED optical behavior, we have run Monte Carlo simulations for a $50 \mu \mathrm{m}$ width LED using the statistical approach we implemented in our SPICE model. We have focused our attention on the spread of $\mathrm{EQE}_{\max }$. Simulations results for 1000 runs are shown on figure 5. The first observation is that the maximum external quantum efficiency shows a Gaussian like distribution (figure 5 (a)) centered on an average value $\left\langle E Q E_{\max }\right\rangle=0.11$. with a spread deviation at $3 \sigma=0.02$. To seek the effect of the random $\mathrm{A}, \mathrm{B}$ and $\mathrm{C}$ value draws on quantum efficiency, all extracted $\mathrm{EQE}_{\max }$ values are presented on three different cloud graphs versus all drawn values of recombination parameters, respectively. Even though, no special correlation between recombination parameters has been used (random draws of $\mathrm{A}, \mathrm{B}$ and $\mathrm{C}$ ), we observe awaited evolutions of $\mathrm{EQE}_{\max }$ versus each related recombination parameter. Indeed, we observe a tendency of having a better quantum efficiency when a higher parameter B value is drawn (figure $5(\mathrm{c})$ ), whatever are $\mathrm{A}$ and $\mathrm{C}$ values. This trend is also observable when A (figure 5 (b)) or $\mathrm{C}$ (figure 5 (d)) show lower values independently of the two other parameters. At last, we think that this kind of cloud representations of Monte Carlo simulations can also help understand and quantify the spread in quantum efficiency of $\mu$ LEDs dedicated for display applications. Indeed high resolution $\mu \mathrm{LED}$ arrays contain a huge number of single $\mu$ LEDs that will have to be controlled 
efficiently by an active matrix which also has its own spread. Therefore an InGaN/GaN LED SPICE model is mandatory to evaluate and design robust $\mu$ LED displays.
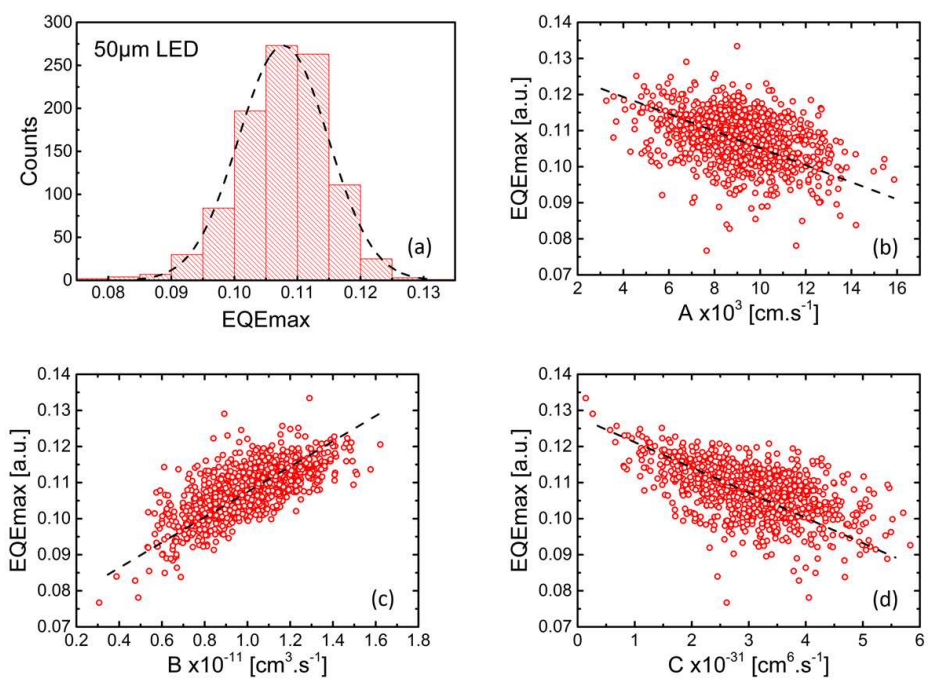

Figure 5. 1000 run Monte Carlo simulation results of a 50 $\mu$ m width LED. (a) Histogram Gaussian like distribution of $\mathrm{EQE}_{\max }$. Cloud graphs in (b), (c) and (d) represent EQEmax versus SRH, radiative and Auger recombination related parameters A, B and $\mathrm{C}$, respectively. Awaited trends of $\mathrm{EQE}_{\max }$ are observed versus each parameter showing the robustness of our implemented statistical approach in the SPICE model.

\section{CONCLUSION}

An InGaN/GaN LED SPICE model taking into account known size effects is demonstrated. A sub-circuit description of the LED permitted an accurate modelling of the electrical behavior. The use of the ABC model recombination related parameters with a simultaneous mathematical resolution during current simulations allowed a precise determination of the external quantum efficiency versus current density. Moreover, a first simple statistical approach based on Gaussian distributions applied to recombination parameters show that this kind of SPICE modelling can help understand and ameliorate pixel designs dedicated to high resolution displays that are aimed to applications for virtual, augmented or mixed reality.

\section{ACKNOWLEGEMENTS}

The authors acknowledge partial fundings from the European Union's Horizon 2020 VOSTARS research and innovation programme under grant agreement No 731974 and H2020 HILICO European project (H2020- JTI-CS2-2016-CFP04-SYS01-03, Grant No. 755497).

\section{REFERENCES}

[1] Olivier, F., Tirano, S., Dupré, L., Aventurier, B., Largeron, C., Templier, F., "Influence of size reduction on the performances of GaN-based micro-LEDs for display application”, J. Lumin., 191, pp.112-116, (2017).

[2] Tian, P., McKendry, J. J. D., Gong, Z., Guilhabert, B., Watson, I. M., Gu, E., Chen, Z., Zhang, G., Dawson, M. D., "Size dependent efficiency and efficiency droop of blue InGaN micro-light emitting diodes", Appl. Phys. Lett., 101, 231110, (2012). 
[3] Badock, T. J., Ali, M., Zhu, T., Pristovsek, M., Oliver, R. A., Shields, A. J., "Radiative recombination mechanisms in polar and non-polar InGaN/GaN quantum well LED structures", Appl. Phys. Lett., 109, 151110, (2016).

[4] Day, J., Li, J., Lie, D. Y. C., Bradford, C., Lin, J. Y., Jiang, H. X., "III-Nitride full-scale high-resolution microdisplays", Appl. Phys. Lett., 99, 031116 (2011).

[5] El-Ghoroury, H. S., Alpaslan, Z. Y., "Quantum Photonic Imager (QPI): A New Display Technology and its Applications", Proc. of the $21^{\text {st }}$ International Display Workshop (IDW'14), Niigata, Japan (2014).

[6] Templier, F., Dupré, L., Tirano, S., Marra, M., Verney, V., Olivier, F., Aventurier, B., Sarrasin, D., Marion, F., Catelain, T., Berger, F., Mathieu, L., Dupont, B., Gamarra, P., "GaN-based Emissive Microdisplays: A very Promising Technology for Compact, Ultra-high Brightness Display Systems”, SID Int. Symp. Digest Tech. Papers, 47(1), pp. 1013-1016, (2016).

[7] Nagel, L. W, Pederson, D. O., "SPICE (Simulation Program with Integrated Circuit Emphasis)", Memorandum No. ERL-M382, University of California, Berkeley, (1973).

[8] Daami, A., Vaillant, J., Gwoziecki, R., Serbutoviez, C., “A complete SPICE subcircuit-based model library for organic photodiodes", Solid-State Electronics, 75, 81-85, (2012).

[9] Cummins, G., Underwood, I., Walton, A., "Electrical characterization and modelling of top-emitting PINOLEDs", Journal of the Society for Information Display, 19, 360-367, (2011).

[10] Lee, S.-G., Hattori, R., "Physics-based OLED analog behavior modeling", Journal of Information Display, 10, 101-106, (2009).

[11] Prajoon, P., Nirmal, D., Anuja Menokey, M., Charles Pravin, J., “A modified ABC model in InGaN MQW LED using compositionally step graded Alternating Barrier for efficiency improvement", Superlattices and Microstructures, 96, 155-163, (2016).

[12] Lin, G.-B., Meyaard, D., Cho, J., Schubert, E. F., Shim, H., Sone, C., “Analytical model for the efficiency droop in semiconductors with asymmetric carrier-transport properties based on drift-induced reduction of injection efficiency”, Appl. Phys. Lett., 100, 161106, (2012).

[13] S. Karpov, "ABC-model for interpretation of internal quantum efficiency and its droop in III-nitride LEDs: a review” Opt. Quantum Electron., Vol. 47, pp.1293-1303, (2015)

[14] Bulashevich, K. A., Konoplev, S. S., Karpov, S. Y., Photonics, 5(4), 41, (2018).

[15] Olivier, F., Daami, A., Licitra, C., Templier, F., "Shockley-Read-Hall and Auger non-radiative recombination in GaN based LEDs: A size effect study", Appl. Phys. Lett., 111, 022104, (2017).

[16] Daami, A., Olivier, F., Dupré, L., Henry, F., Templier, F., "Electro-optical size-dependence investigation in GaN micro-LED devices”, SID Int. Symp. Digest Tech. Papers, 49(1), 790-793 (2018).

[17] Nickalls, R. W. D., (1993), “A new approach to solving the cubic: Cardan's solution revealed” The mathematical Gazette, 77(480), 354-359, (1993).

[18] Zucker, I. J., "The cubic equation: a new look at the irreducible case”, The Mathematical Gazette, 92(52), 264268, (2008). 